\title{
Dynamic Analysis and Simulation on Bionics Quadruped Robot
}

\author{
Jinrong Zhang* Jianhua Zhang and Chenxi Wang
}

\author{
Chang'an University, Xi'an, Shaanxi, 710064, China
}

\begin{abstract}
The structural framework of quadruped robot was built based on the bionics. Then, lead out the dynamic equations of the simplified structure of the multiple rigid body system using Lagrange method considering the structural characteristic of quadruped robot. At the same time, the virtual prototype of quadruped robot was established and simulated using ADAMS with the planned gait. The simulation results verified the validity of mathematical modeling and the feasibility of the structure design, and it provided useful data information for the subsequent study to enhance the quality of control.
\end{abstract}

Keywords: Bionics, Quadruped robot, Dynamic analysis, Simulation.

\section{INTRODUCTION}

Compared with the traditional wheeled and tracked robot, quadruped robot has the strong adaptability to environment and flexibility which can enter the relatively narrow space, pass through obstacles, ascend and descend the stairs and slopes, move on rough ground and has good prospects in emergency rescue and disaster relief, mine clearance, exploration, entertainment, military, and so on.

As the key issue, the dynamics of a quadruped robot is the foundation to realize the high precision control and mechanical design. But the quadruped robot is an active mechanical device with a great many degrees of freedom in the walking structure, and each joint can drive separately. From a control theory standpoint, it is a complex dynamic coupling system, which has the significantly nonlinear and complicated mathematical model $[1,2]$. In this paper, the quadruped robot was dynamically modeled and simulated, to provide a mathematical model for the subsequent robot control algorithm, and a theoretical basis for the robot's structure optimization design and the selection of joint driving motors and reducers.

\section{STRUCTURE DESIGN}

Dogs with four legs (shown in Fig. (1).) have more degrees of freedom, which mean they have more skeleton and skeleton connection forms with multi degree of freedom to help them move flexibility [3]. At present, it's unrealistic to completely copy an animal. So with the research on the robots' movement mechanism, we can simplify the structure on the basis of dogs' biological structure, combine the skeletons with small amplitude, leave out the skeletons with unobvious motion, and decrease the members and degrees of freedom of limbs as much as possible to achieve a simple structure which can meet the motion requirements $[4,5]$.

*Address correspondence to this author at the Chang'an University, Xi'an, Shaanxi, 710064, China; Tel: 13991995604;

E-mail: 17672467@qq.com

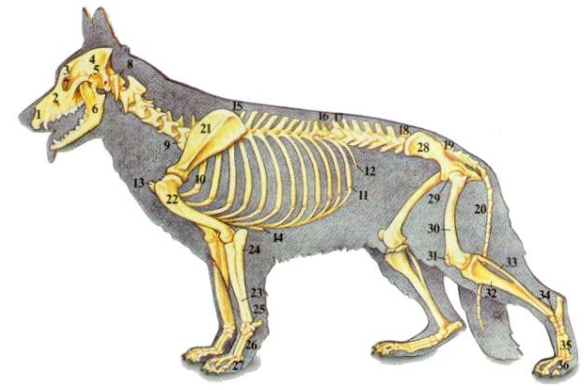

Fig. (1). The German Shepherd Dog Skeletal Structure.

The simplified structure is shown in Fig. (2), the model structure is divided into body, thighs and calves, and they are connected with each other through joints. Hip joints are respectively less connected with the body, and they have rotational degrees of freedom around the forward axis and normal axis of the plane; the knee joints are connected with the thighs, and they have rotational degrees of freedom around the normal axis of the plane.

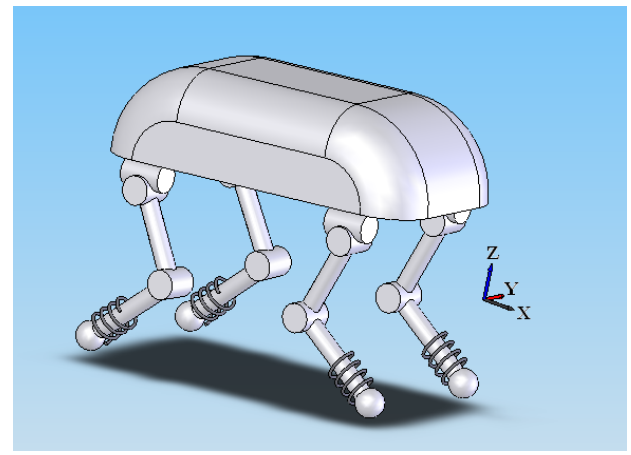

Fig. (2). Structure Model of Bionic Quadruped Robot.

The basic parameters of the quadruped robot structure are shown in Table 1:

\section{DYNAMIC MODELING}

The methods commonly used in the robot dynamics analysis are the Newton Euler equation and Lagrange. La- 
Table 1. Basic Parameters of Robot.

\begin{tabular}{|c|c|c|c|c|c|c|}
\hline Parameters & Length & Width & Height (stand) & Thigh Length & Calf Length & Weight \\
\hline \hline value & $550 \mathrm{~mm}$ & $230 \mathrm{~mm}$ & $520 \mathrm{~mm}$ & $200 \mathrm{~mm}$ & $200 \mathrm{~mm}$ & $25.4 \mathrm{~kg}$ \\
\hline
\end{tabular}

grange is a kind of functional balance method which doesn't need internal force, just speed. It is a direct and simple method. In this paper, Lagrange was used to analyze and solve the dynamic equation of the walking foot with three degrees of freedom.

Body structure of quadruped robot is shown in Fig. (3). The side swing joint rotates in YOZ plane. $m_{1}, m_{2}$ and $m_{3}$ are respectively mass of the side swing, mass of the thigh and mass of the calf. $\theta_{1} 、 \theta_{2}$ and $\theta_{3}$ are the joint angles while $g$ is the acceleration of gravity.

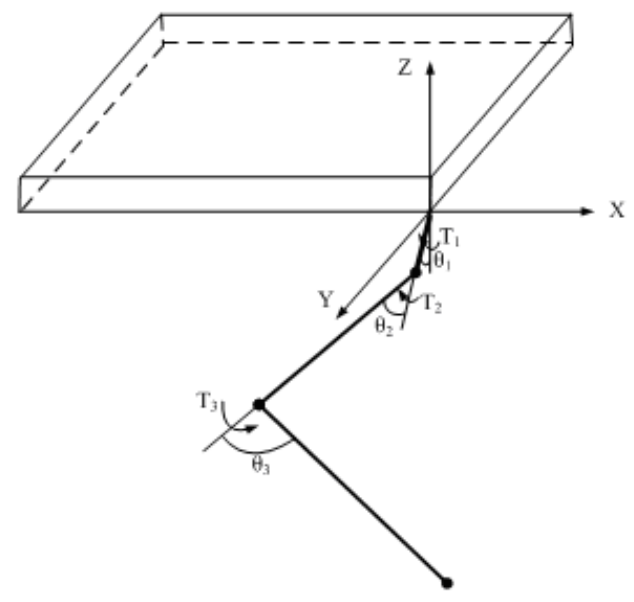

Fig. (3). Limb Structure of Quadruped Robot.

Lagrange dynamics equation of mechanical system is:

$\mathrm{T}_{\mathrm{i}}=\frac{d}{d t} \frac{\partial E_{K}}{\partial \dot{q}_{i}}-\frac{\partial E_{K}}{\partial q_{i}}+\frac{\partial E_{P}}{\partial q_{i}}$

Where,

$E_{K}, E_{P}, q_{i}, \dot{q}_{i}$ and $T_{i}$ being the total kinetic energy of the system, the total potential energy of the system, the angle coordinate of the joint, the angular velocity of the joint and the joint torque respectively. Linear and angular velocities of the center of mass of the connecting rod $i$ were determined as:

$\mathrm{v}_{\mathrm{i}}=J_{L 1}^{i} \dot{q}_{1}+\cdots+J_{L i}^{i} \dot{q}_{i}=J_{L}^{i} \dot{q}$

$\omega_{\mathrm{i}}=J_{A 1}^{i} \dot{q}_{1}+\cdots+J_{A i}^{i} \dot{q}_{i}=J_{A}^{i} \dot{q}$

Where, $J_{L i}$ and $J_{A i}$ are the Jacobi matrixes associated with a translational and rotational velocity of the center of gravity of the connecting rod $i$. When $|J|=0$, robot's position become singular state.
Translational energy of the system can be defined as:

$E_{K 1}=\frac{1}{2} \sum_{i=1}^{n} m_{i} \dot{q}^{T} J_{L}^{(i) T} J_{L}^{(i)} \dot{q}$

Kinetic rotation energy of the system can be defined as:

$$
E_{K 2}=\frac{1}{2} \sum_{i=1}^{n} \dot{q}^{T} J_{A}^{(i) T} I_{i} J_{A}^{(i)} \dot{q}
$$

The total kinetic energy of the system is defined as the sum of the translational energy and rotational energy [6], which can be defined as:

$$
\begin{aligned}
E_{K}= & E_{K 1}+E_{K 2} \\
& =\frac{1}{2} \sum_{i=1}^{n}\left(m_{i} \dot{q}^{T} J_{L}^{(i) T} J_{L}^{(i)} \dot{q}+\dot{q}^{T} J_{A}^{(i) T} I_{i} J_{A}^{(i)} \dot{q}\right)
\end{aligned}
$$

Where $H$ can be obtained by the following:

$$
H=\sum_{i=1}^{n}\left(m_{i} J_{L}^{(i) T} J_{L}^{(i)}+J_{A}^{(i) T} I_{i} J_{A}^{(i)}\right)
$$

The total potential energy of the system is:

$$
E_{p}=\sum_{i=1}^{n} m_{i} g^{T} r_{0, i}
$$

Where,

$r_{0, i}$ is the position of the center of mass of the connecting rod $i$ in the reference frame.

According to formulate (1), (6) and (8), the torque of each joint can be defined as:

$$
\mathrm{T}_{\mathrm{i}}=\sum_{j=1}^{n} H_{i j} \ddot{q}_{j}+\sum_{j=1}^{n} \sum_{k=1}^{n} h_{i j k} \dot{q}_{j} \dot{q}_{k}+\sum_{j=1}^{n} m_{j} g^{T} J_{L i}^{j}
$$

Where,

$$
h_{i j k}=\frac{\partial H_{i j}}{\partial q_{k}}-0.5 \frac{\partial H_{j k}}{\partial q_{i}}
$$

\section{SIMULATION OF THE MODEL}

\subsection{Simulation Assumptions}

Spatial structure often moves non-continuously in practical application. For example, the quadruped robot foot landing the ground and the change of the motion state of the system meant that two or more objects collided in the same or different multiple body system. The physical collision pro- 
cess was very complex, often accompanied with a large impact force. At the same time, the collision force can stimulate the structure of a high order model, which definitely increased the complexity of the system dynamic response, and put forward a higher requirement for the control system.

The following assumptions were applied in the research to simplify the problem:

(1) The collision process happened instantly and collision objects were regarded as rigid bodies;

(2) The surface of the collision can be considered as a point which didn't change in the collision process.

(3) $e$ is the restitution coefficient which was the ratio of the completely inelastic collision impulse and of the collision impulse in the compression phase. It is a completely elastic collision when $e=1$ and a completely inelastic collision when $e=0 \quad \mathrm{e}=0$. Assuming that there is no change of restitution coefficient in the collision process.

\subsection{Establishment of Simulation Model}

Virtual prototype modeling was achieved in the SolidWorks environment. The prototype model data were leading into ADAMS through the Parasolid interface form. In the ADAMS environment, the body is set for aluminum material and supporting ground is set for a steel plane. Set the constraint according to the degrees of freedom, set the parameters such as contact, friction, elastic modulus by seeking table and add the drives to active degrees of freedom. After that, simulation can be carried to do the analysis by setting different parameters.

The virtual prototype model is shown in Fig. (4).

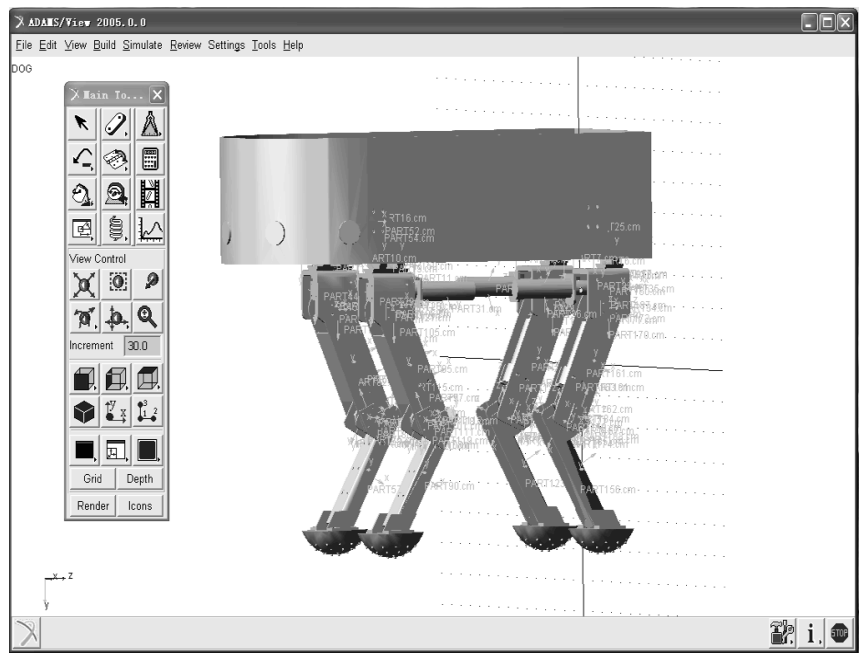

Fig. (4). Virtual Prototype Model in ADAMS/View.

The flowchart of simulation is shown in Fig. (5).

\subsection{Simulation Result}

For the trot [7] gait, the motions of the two diagonal legs were exactly symmetrical. Take the right front leg and left back leg for example, their driving torques of hip and knee are shown as Figs. (6-9).

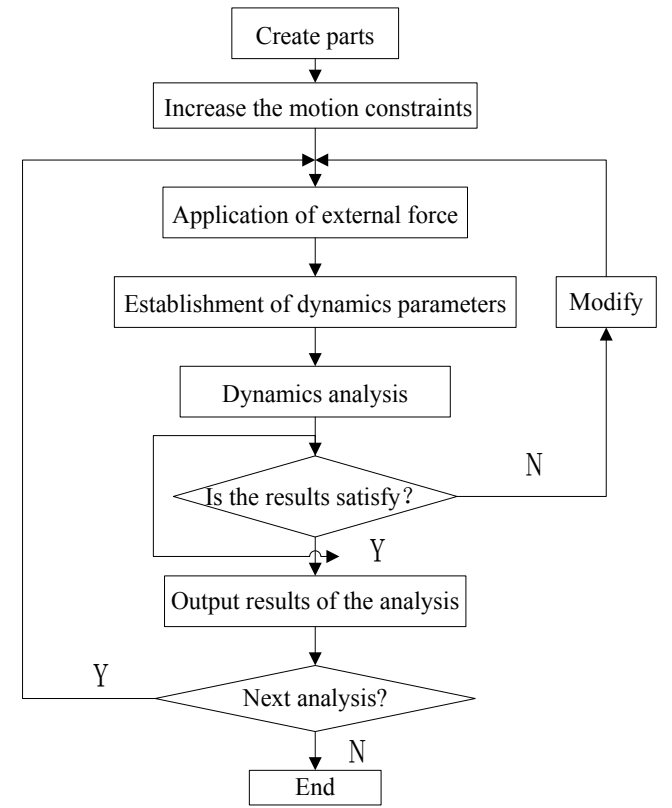

Fig. (5). Flowchart of Dynamics Simulation.

It can be seen from the Lagrange dynamics equations of quadruped robot (Formula (9)) that the torque was connected with model structure's parameters, angular velocity and angular acceleration.

The curves (Figs. (6-9)) of torque with different angular velocity and acceleration can be simulated with the unchangeable structure parameters. The following results can be analyzed from the curves:

1) Driving force of the hip and knee joints is larger in the support phase than in the swing phase;

2) The unbalanced driving torque of the hip and knee joints mainly appeared at the end point in swing phase. At that point, $\theta_{1}=0, \theta_{2}=12.32^{\circ}, \theta_{3}=0$ and the Jacobi matrix $|J|=0$, robot was in Jacobi singular configurations state. So there will appear a sudden change (peak of curve) in the simulation diagram;

3) Except for Jacobi singular state, the driving torque of the hip joint was mainly concentrated in the range of 25 $N \bullet m$, while the driving torque of the knee joint was mainly concentrated in the range of $50 \mathrm{~N} \cdot \mathrm{m}$.

\section{CONCLUSION}

A dynamic model of quadruped robot was set up by application of Lagrange's theory, which provided a theoretical basis of robots structure optimization and provided a mathematical model for a quadruped robot control algorithm design. The virtual prototype of quadruped robot was built to get the driving torque of each leg of the hip and knee using the dynamic simulation software. The simulation results can provide the basis for the selection of drive motor and reducer. The correctness and the rationality of the structure design of mathematical modeling were also verified. 


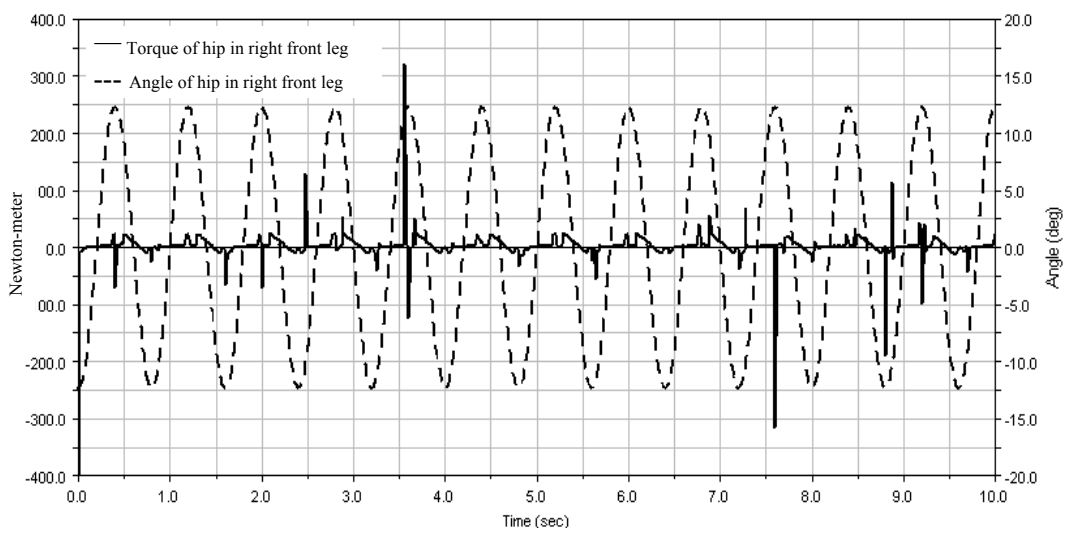

Fig. (6). Torque and Angle of Hip in Right Front Leg.

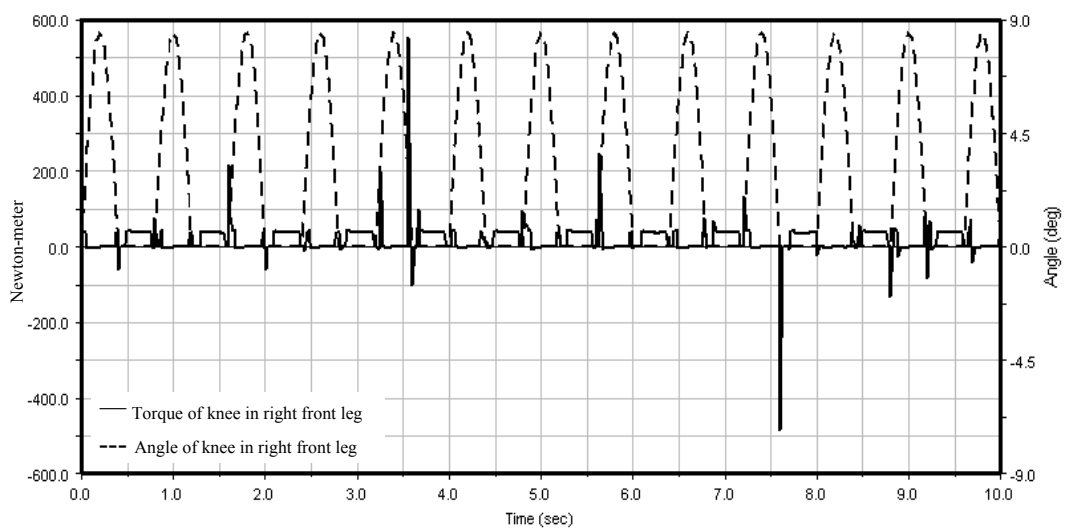

Fig. (7). Torque and Angle of Knee in Right Front Leg.

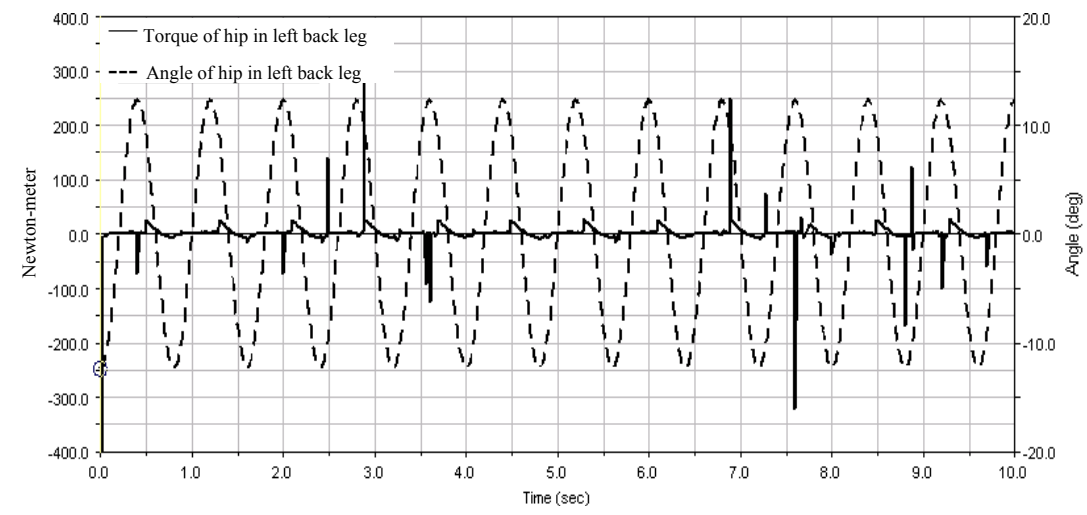

Fig. (8). Torque and Angle of Hip in Left Back Leg.

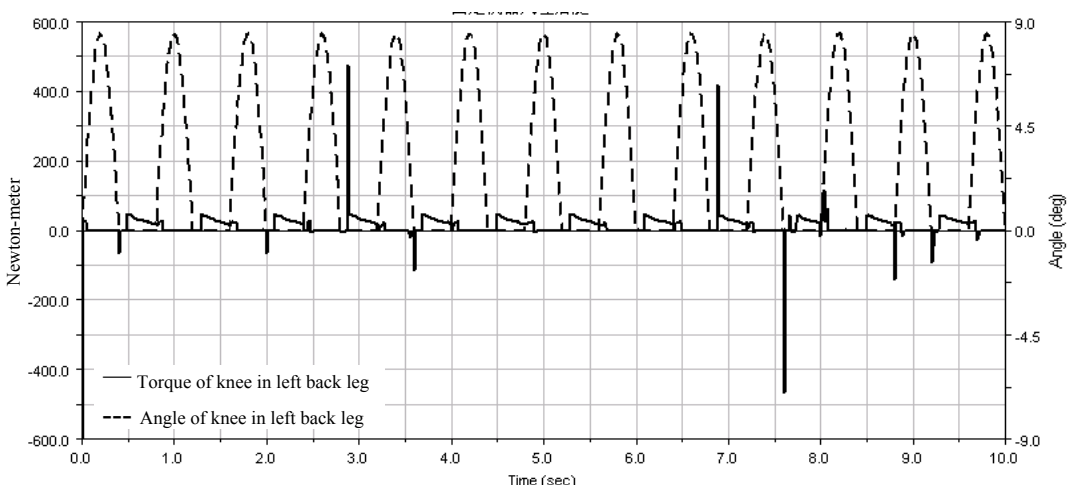

Fig. (9). Torque and Angle of Knee in Left Back Leg. 


\section{CONFLICT OF INTEREST}

The authors confirm that this article content has no conflict of interest.

\section{ACKNOWLEDGEMENTS}

Declared none.

\section{REFERENCES}

[1] T. Otani, T. Urakubo, S. Maekawa, and H. Tamaki, "Position and attitude control of a spherical rolling robot equipped with a gyro," IEEE International Workshop on Advanced Motion Control, pp. 416-421, 2006.
[2] W.Schiehlen, "Multi-body Systems Handbook," Berlin: SpringerVerlag, pp. 13 69, 1990.

[3] T. Ho, S. Choi, and S. Lee, "Development of a Biomimetic Quadruped Robot," Journal of Bionic Engineering, vol. 4, pp. 193-199, 2007.

[4] R. Playter, M. Buehler, and M. Raibert, "BigDog. Proceedings of SPIE," Unmanned Systems Technology VIII, vol. 6230, 2006.

[5] J.R. Rebula, P.D. Neuhaus, B.V. Bonnlander, and M.J. Johnson, "A controller for the little dog quadruped walking on rough terrain, In: Proceedings of the IEEE International Conference on Robotics and Automation (ICRA), pp.1467-1473, 2007.

[6] M. Wang, L. Wang, Q. Meng, P. Yuan, and Y. Yu, "Dynamic modeling and simulation of biomimetic crablike robot with ADAMS," Journal of Harbin Engineering University, vol. 4, 2003.

[7] X. Zhang, "Biological-inspired Rhythmic Motion \& Environmental Adaptability for Quadruped Robot,” Ph.D. thesis. Tsinghua University, vol. 4, 2004

(C) Zhang et al.; Licensee Bentham Open.

This is an open access articles licensed under the terms of the Creative Commons Attribution-Non-Commercial 4.0 International Public License (CC BY-NC 4.0) (https://creativecommons.org/licenses/by-nc/4.0/legalcode), which permits unrestricted, non-commercial use, distribution and reproduction in any medium, provided that the work is properly cited. 